\title{
Ovariohisterectomia videoassistida com dois portais para o tratamento de piometra em cadela
}

\author{
Videoassisted ovariohysterectomy with two portals for pyometra's treatment in a bitch
}

\author{
Rogério Luizari Guedes ${ }^{\text {* }}$ Caroline Posser Simeoni ${ }^{\mathrm{II}}$ Marcella Teixeira Linhares ${ }^{\mathrm{II}}$ Ialo Ferro de Castro \\ Junior ${ }^{\text {II }}$ Thiago de Oliveira Cunha ${ }^{\text {II }}$ Fernando Wiecheteck de Souza ${ }^{\text {I }}$ Ney Luis Pippi ${ }^{I I}$
}

\section{- NOTA-}

\section{RESUMO}

O presente trabalho descreve o tratamento de piometra em uma cadela submetida à ovariohisterectomia videoassistida por meio de dois portais. O acesso da cavidade abdominal ocorreu através de duas incisões de $1 \mathrm{~cm}$ em linha média, sendo os ovários fixados na parede abdominal por um reparo transcutâneo e, após a visualização do leito vascular, iniciou-se a colocação de clipes de titânio para hemostasia com posterior secção dos cotos ovarianos. A incisão pré-púbica foi ampliada, sendo os ovários e útero exteriorizados e o corpo uterino ligado pelo método convencional de pediculação. $O$ tratamento de piometra videoassistido com dois portais foi realizado em tempo cirúrgico hábil, quando comparado aos observados em procedimentos laparoscópicos na literatura, sem complicações e com perda sanguínea mínima.

Palavras-chave: canino, laparoscopia, videocirurgia, hiperplasia endometrial cística.

\section{ABSTRACT}

This report describes pyometra's treatment in a bitch which was submitted to video-assisted ovariohysterectomy by two portals. The approach into abdominal cavity was carried through two midline incisions of $1 \mathrm{~cm}$ each, and the ovaries fixed in the abdominal wall by a transcutaneous suspension suture and after the identification of the vascular bed, titanium clips were placed for hemostasis with subsequent section of the ovarian stumps. The pre-pubic incision had to be enlarged to remove the ovaries and uterine horns, ligating the uterine body externally by the conventional technique. The video-assisted

\begin{abstract}
treatment of pyometra with two portals was performed in able surgical time when compared with similar procedures described, without complications and minimal blood loss.
\end{abstract}

Key words: canine, laparoscopy, video surgery, cystic endometrial hyperplasia.

A cirurgia laparoscópica está ganhando importância em medicina veterinária, permitindo a realização de intervenções por procedimentos menos invasivos com decréscimo da dor pós-operatória e retorno mais rápido às atividades normais (MINAMI et al.,1997; MCCLARAN \& BUOTE, 2009). Por promover incisões cirúrgicas menores, limita o trauma dos tecidos, minimiza as chances de deiscência de feridas e, no caso de coagulopatias, a laparoscopia diminui o sangramento parietal (COLLARD \& VIGUIER, 2008).

Considerando a elevada frequência de piometra em cadelas e que a ovariohisterectomia (OSH) representa um dos procedimentos mais comumente realizados na medicina veterinária (GROOTERS, 1994), este trabalho relata um caso clínico de diagnóstico e tratamento de piometra por meio de uma técnica de OSH vídeoassistida por dois portais em uma paciente que foi encaminhada para o procedimento eletivo.

IPrograma de Pós-graduação em Medicina Veterinária, Universidade Federal de Santa Maria (UFSM), 97105-900, Santa Maria, RS, Brasil. E-mail: rogerioguedes@veterinario.med.br. *Autor para correspondência.

IPrograma de Pós-graduação em Medicina Veterinária, UFSM, Santa Maria, RS, Brasil.

IIIDepartamento de Clínica de Pequenos Animais, UFSM, Santa Maria, RS, Brasil. 
Uma cadela adulta sem raça definida com peso corporal de $20 \mathrm{~kg}$ passou por exame clínico e laboratorial (hemograma e avaliações de plaquetas, albumina, alanina-amino-transferase e creatinina), com valores dentro da normalidade. A paciente recebeu medicação pré-anestésica com maleato de acepromazina $\left(0,03 \mathrm{mg} \mathrm{kg}^{-1}\right.$, SC) e cloridrato de tramadol $\left(5 \mathrm{mg} \mathrm{kg}^{-1}\right.$, SC). Após 15 minutos, foi induzida à anestesia geral com propofol (3mg kg-1, SC), mantida a $1,8 \% \mathrm{~V}$ de isoflurano expirado e ventilação mecânica ciclada por pressão (15mmHg). Foram aplicados, pela via epidural, cloridrato de lidocaína $2 \%$ com vasoconstritor ( $1 \mathrm{ml} 4 \mathrm{~kg}^{-1}$ ) e cloridrato de morfina $\left(0,1 \mathrm{mg} \mathrm{kg}^{-1}\right)$. A fluidoterapia de manutenção foi realizada com solução Ringer com lactato $\left(10 \mathrm{ml} \mathrm{kg}^{-1} \mathrm{~h}^{-1}\right)$ e a profilaxia antimicrobiana com cefalotina (30 $\mathrm{mg} \mathrm{kg}^{-1}, \mathrm{IV}$ ). Iniciou-se a intervenção cirúrgica em decúbito dorsal, realizando uma incisão de pele de aproximadamente $1,2 \mathrm{~cm}$ sobre a cicatriz umbilical para a introdução do primeiro trocarte/cânula (11mm de diâmetro) pelo método aberto. Confirmada a entrada na cavidade, foi iniciado o pneumoperitônio, com $\mathrm{CO}_{2}$ medicinal sob pressão de $12 \mathrm{mmHg}$ e fluxo de 2L min $^{-1}$. Um endoscópio de $10 \mathrm{~mm}$ foi inserido através da cânula e, neste momento, foi verificado que o útero apresentava dimensões aumentadas, com certa flacidez e presença de conteúdo. A seguir, foi posicionado o segundo portal $(11 \mathrm{~mm})$, em linha média na região prépúbica, e a paciente foi posicionada em decúbito lateral direito para a visualização do ovário contralateral.

O ovário foi apreendido por uma pinça Kelly e elevado até a parede abdominal esquerda, sendo aplicado um reparo transcutâneo com agulha traumática $3 / 8$ e fio seda $n^{\circ} 0$, através do mesométrio em região de tubas uterinas. Pelo peso excessivo do corno uterino, foi necessário outro reparo percutâneo (Figura 1A) para visualizar adequadamente o complexo arteriovenoso ovariano (CAVO). Deu-se início à hemostasia através de clipes de titânio tamanho médio-grande e clipador de $10 \mathrm{~mm}$ de diâmetro. Sua aplicação foi intercalada, sendo um proximal e outro distal, seguida da secção parcial com tesoura de Metzenbaum (Figura 1B), para permitir o avanço do clipador no tecido em questão. $\mathrm{O}$ mesmo processo foi realizado para o ovário direito. $\mathrm{O}$ número total de clipes utilizados foi de 41 , sendo 20 para o ovário esquerdo e 21 para o direito (Figura 1C), permanecendo implantados 16 clipes na paciente (oito em cada lado).

Após o fim da hemostasia do CAVO direito, o ovário foi apreendido através do ligamento suspensor e tracionado junto ao portal caudal, sendo necessária sua ampliação em aproximadamente $1,5 \mathrm{~cm}$, criando uma ferida cirúrgica de aproximadamente $3,0 \mathrm{~cm}$ para a exposição de corpo e cornos uterinos (Figura 1D), que apresentavam cerca de $4 \mathrm{~cm}$ de diâmetro. O corpo uterino foi ligado pela técnica convencional de pediculação, com fio mononáilon 2-0 e ligadura em “8”, sendo devolvido à cavidade. Nova exploração laparoscópica foi realizada e, após se confirmar a ausência de sangramento, o pneumoperitônio foi desfeito. A síntese das feridas foi realizada com fio monofilamentar de náilon em colchoeiro em cruz (2-0) na musculatura, mesmo padrão (3-0) no subcutâneo, colchoeiro horizontal e mesmo fio para pele.

O tempo cirúrgico total foi de 158 minutos. A remoção do sangue proveniente das incisões na pele e tecido subcutâneo assim como do procedimento de OSH foi realizada com gazes laparoscópicas, sendo que o sangramento durante a OSH ocorreu principalmente durante a hemostasia dos CAVOs, totalizando uma perda de aproximadamente $43,9 \mathrm{ml}$. Após o final do procedimento, o útero removido foi puncionado, drenando conteúdo purulento, confirmando a suspeita diagnóstica de piometra. O tratamento pós-operatório consistiu na administração de meloxicam $\left(0,2 \mathrm{mg} \mathrm{kg}^{-1}\right.$, SC, SID) e cloridrato de tramadol ( $3 \mathrm{mg} \mathrm{kg}^{-1}$, SC, TID) por três dias, não havendo complicações até o momento da retirada dos pontos, sete dias após o procedimento cirúrgico.

A utilização de somente dois portais para a realização da OSH no presente relato, segue o princípio proposto para aquelas eletivas relatadas por DEVITT et al. (2005), porém, neste caso, devido ao uso de endoscópio sem canal de trabalho, a introdução do segundo portal ocorreu imediatamente após o posicionamento do primeiro, como realizado por BRUN et al. (2008). O uso da sutura de suspensão transabdominal do pedículo ovariano o manteve exposto sem necessidade de portais adicionais (ROSIN et al., 2001). A troca de decúbito realizada durante a cirurgia facilitou a visualização do útero e ovários e a fixação das bolsas ovarianas na parede abdominal para hemostasia e secção do CAVO, a partir do deslocamento do baço e alças intestinais, maximizando a exposição das estruturas envolvidas na OSH (DEVITT et al., 2005). Os autores acreditam que o posicionamento adequado da fixação é o grande responsável pelo sucesso na elevação e sustentação dos ovários e cornos uterinos, porém, a condição geral do útero deve ser avaliada antes de se realizar esta etapa, uma vez que a friabilidade ocorrida pela distensão e o peso poderiam ocasionar a sua ruptura.

Em relação a hemorragias, MALM et al. (2004) relatam ausência dela em 60\% das OSHs laparoscópicas eletivas em animais hígidos $(n=15)$, sangramento discreto em cerca de $27 \%$ e sangramento acentuado em 13,3\%. Esses últimos dois resultados 


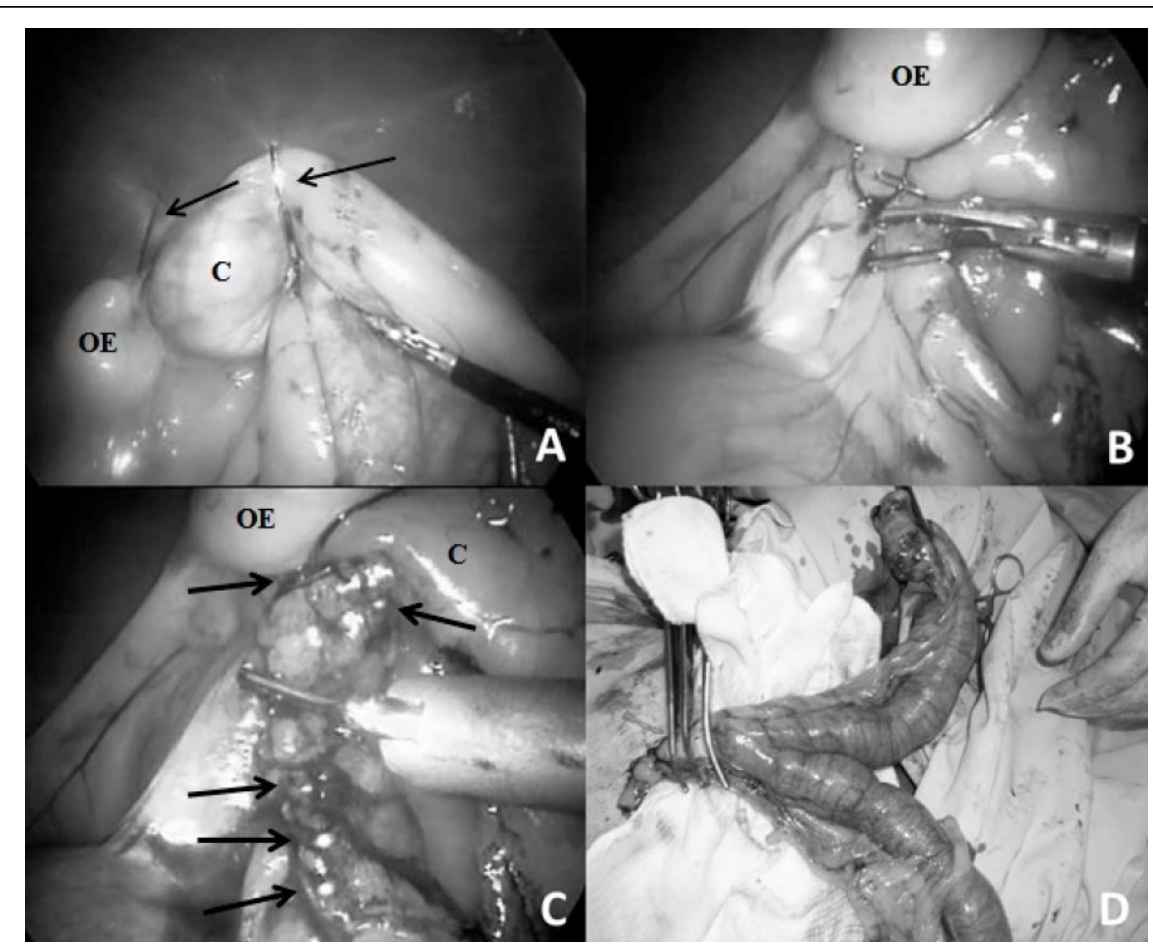

Figura 1 - Etapas da OSH videoassistida. Em A, elevação do ovário esquerdo (OE) e corno uterino (C) por duas suturas de fixação (setas) para visualização do CAVO; em B, a secção parcial do CAVO e ligamento largo uterino após aplicação de clipes proximal e distal; C demonstra a grande quantidade de clipes utilizados para hemostasia durante o avanço da aplicação do clipador; em $\mathrm{D}$, aspecto de útero e ovários exteriorizados pelo acesso pré-púbico ampliado.

ocorreram devido à lesão de ramo da artéria uterina esquerda e corroboram a perda de sangue verificada neste estudo, em que o sangramento dos CAVOs representou menos de $3 \%$ do volume sanguíneo total da paciente. Há diferença significativa de perda sanguínea quando comparadas a OSH aberta e a videoassistida com quatro portais, com menor grau de sangramento nos animais submetidos à cirurgia laparoscópica (MALM et al., 2004).

A similaridade do número de clipes aplicados no CAVO direito e esquerdo, respectivamente 21 e 20, demonstra uniformidade na técnica. Isso pode ser explicado pelo tamanho aumentado do útero, intensa vascularização causada pela piometra e grande deposição de tecido adiposo nos ligamentos largos uterinos, também sendo necessário o uso de clipes para suas hemostasias, uma vez que dificultavam a visualização dos pedículos ovarianos. Os autores acreditam que a presença de mais portais poderiam ter reduzido o número de clipes, assim como o uso de clipes maiores, sendo que MALM et al. (2004) e SCHIOCHET et al. (2009) relatam a utilização de apenas 6 clipes médio-grandes nos vasos dos pedículos ovarianos, no entanto os animais do primeiro estudo não apresentavam nenhuma alteração uterina e a hemostasia foi associada à eletrocauterização durante a secção dos ligamentos largo uterinos e de alguns pequenos vasos dos pedículos ovarianos, enquanto que, no segundo trabalho, os procedimentos foram realizados em gatas.

A variação de tempos cirúrgicos pode ser influenciada por alguns fatores como o método de hemostasia utilizado, o grau de complicação da cirurgia, a experiência do cirurgião e os estados corporal e fisiológico do animal submetido à cirurgia (VAN GOETHEM et al., 2003; DUTTA et al., 2010). Apesar do tempo de $158 \mathrm{~min}$ para este procedimento superar os 85min relatados por COLLARD \& VIGUIER (2008), enquadrou-se na variação de tempo encontrada no estudo de DAVIDSON et al. (2004), em que as OSHs eletivas laparoscópicas variaram entre 47 e 175min. Sabendo-se que alterações uterinas contribuem para aumentar o tempo cirúrgico e que a cirurgia laparoscópica reduz o trauma provocado nos tecidos e o escore de dor no pós-operatório, com o animal se recuperando mais rápido (MINAMI et al., 1997; 
DEVITT et al., 2005; MCCLARAN \& BUOTE, 2009), pode-se considerar que este caso manteve-se dentro de um tempo aceitável para o tratamento de piometra, com recuperação adequada do paciente e sem complicações pós-operatórias. Porém, os autores consideram que, em casos pré-diagnosticados, a opção de cirurgia laparoscópica ocorreria após a avaliação de fatores que poderiam interferir nos riscos e tempo cirúrgico, incluindo o estado geral e porte do paciente e tamanho de cornos uterinos.

A necessidade de se ampliar a incisão prépúbica associada à flacidez dos cornos permitiu a passagem deles sem rupturas durante sua exteriorização. DAVIDSON et al. (2004) também realizaram ampliação do portal para evitar a tração excessiva do útero. Estes autores comentam que animais jovens podem ter útero e ovários friáveis, podendo ser rompidos (ou lacerados) por manobras cirúrgicas pouco delicadas durante sua remoção.

A técnica videoassistida através de dois portais foi exequível no tratamento para piometra no presente caso, apesar de sua real aplicação para tal afecção não estar estabelecida por completo. O procedimento pode ser realizado em tempo cirúrgico equivalente quando comparado aos observados em outros métodos laparoscópicos na literatura, embora seja necessário constante treinamento da equipe para que seja reduzido.

\section{REFERÊNCIAS}

BRUN, M.V. et al. Ovariosalpingohisterectomia vídeo-assistida com dois portais em cadela - Relato de três casos. In: CONGRESSO REGIONAL DE VIDEOCIRURGIA, 11., 2008, Búzios, RJ. Revista Brasileira de Videocirurgia, v.5, n.1, p.58, 2008.

COLLARD, F.; VIGUIER, E. A pyometra managed by laparoscopic ovariohysterectomy in a dog. Revue de Médecine Vétérinaire, v.159, n.12, p.624-627, 2008.

DAVIDSON, E.B. et al. Comparison of laparoscopic ovariohysterectomy and ovariohysterectomy in dogs. Veterinary Surgery, v.33, p.62-69, 2004.
DEVITT, C.M. et al. Duration, complications, stress, and pain of open ovariohysterectomy versus a simple method of laparoscopic-assisted ovariohysterectomy in dogs. Journal of the American Veterinary Medical Association, v.227, p.921-927, 2005.

DUTTA, A. et al. Evaluation of different laparoscopic sterilization techniques in a canine birth control program. Turkish Journal of Veterinary and Animal Sciences, v.34, n.4, p.393-402, 2010.

GROOTERS, A.M. Diseases of the ovaries and uterus. In: BIRCHARD, S.J.; SHERDING, R.G. Saunders manual of small animal practice. Ohio: Saunders, 1994. p.613-632.

MALM, C. et al. Ovário-histerectomia: estudo experimental comparativo entre as abordagens laparoscópica e convencional na espécie canina. Intra-operatório-I. Arquivo Brasileiro de Medicina Veterinária e Zootecnia, v.56, n.4, p.457-466, 2004.

McCLARAN, J.K.; BUOTE, N.J. Complications and need for conversion to laparotomy in small animals. Veterinary Clinics of North America: Small Animal Practice, v.39, p.941951, 2009. Disponível em: <http://www.sciencedirect.com/science/ article/pii/S01955616 09000680>. Acesso em: 15 abr. 2011. doi: $10.1016 /$ j.cvsm.2009.05.003.

MINAMI, S. et al. Successful laparoscopic assisted ovariohysterectomy in two dogs with pyometra. Journal of Veterinary Medical Science, v.59, p.845-847, 1997.

ROSIN, D. et al. Laparoscopic transabdominal suspension sutures. Surgical Endoscopy, v.15, p.761-763, 2001. Disponível em: <http://www.springerlink.com/content/ epx55cktdxvec4hy/fulltext.pdf>. Acesso em: 15 abr. 2011. doi: $10.1007 / \mathrm{s} 004640080101$.

SCHIOCHET, F. et al. Ovário-histerectomia laparoscópica em felinos hígidos: estudo comparativo de três métodos de hemostasia. Arquivo Brasileiro de Medicina Veterinária e Zootecnia, v.61, n.2, p.369-377, 2009. Disponível em: <http://dx.doi.org/10.1590/S0102-09352009000200013>. Acesso em: 15 abr. 2011. doi: 10.1590/S010209352009000200013.

VAN GOETHEM, B.E.B.J. et al. Monopolar versus bipolar electrocoagulation in canine laparoscopic ovariectomy: a nonrandomized, prospective, clinical trial. Veterinary Surgery, v.32, p.464-470, 2003. 